\section{Task-Dependent Synergies for Motion Planning of an Anthropomorphic Dual-Arm System}

\author{
Néstor García, Raúl Suárez and Jan Rosell
}

\begin{abstract}
The paper deals with the problem of motion planning for anthropomorphic dual-arm robots. It introduces a measure of the similarity of the movements needed to solve two given tasks. Planning using this measure to select proper arm synergies for a given task improves the planning performance and the resulting plan.
\end{abstract}

Index Terms-Path Planning for Manipulators, Humanoid Robots, Synergies, Dimensionality Reduction.

\section{INTRODUCTION}

Current technology has allowed the development of anthropomorphic robots with the capacity to perform movements that mimic human ones, but, nevertheless, the complexity of planning useful movements for some given tasks within a reasonable time and preserving the human appearance is still an open field of research. The problem of motion planning in robotics was addressed with different approaches [1], and, currently, among the most used planners for systems with high number of degrees of freedom (DOF) are the sampling-based planners [2] like, for instance, the Probabilistic Road Map planners (PRM) [3] or the Rapidly-exploring Random Trees planners (RRT) [4].

When the robotic system is anthropomorphic, the use of real movements of a human being as a reference is common, either with the goal of a direct on-line teleoperation of the robotics arms [5], or with the aim of analyzing them and getting some valuable information to be applied later in the planning phase, allowing a lower planning complexity and/or looking for more human-like movements. This latter approach was especially applied in planning motions and grasp configurations of anthropomorphic robotic hands, which are devices with many DOF (typical anthropomorphic robotic hands have four or five fingers with three or four DOF per finger). The basic idea is to establish couplings between the DOF of the robotic hand fingers equivalent to those existing in the human hand. Relevant pioneering works dealt with the grasping problem, analyzing the correlations of finger joints when the hand was grasping an object, and called them "hand postural synergies" [6]. Other works used the same concept to find pre-grasp hand configurations [7], and called "eigengrasp" to each independent hand movement involving correlated movements of all the joints. These works allowed a reduction of the grasp space down to a 2-dimensional space. Later, a compliant model for synergies, called "soft synergies", was introduced and used in the selection of grasping forces, in their control, and in the control of the motion of the grasped object [8], [9]. The main motivation of these works was the grasp synthesis, but there were also works using the same concept to do motion planning trying to mimic human hand postures [10], [11], in this case the correlations between the finger joints were used to determine movement directions so they were called "principal motion directions" (PMDs). As a difference with grasp synthesis, motion planning requires the knowledge of the finger correlations when the fingers are freely moved in the hand workspace without external constraints [12].

The previous discussion on the use of hand synergies to reduce the motion-planning complexity as well as to look for robot movements

The authors are with the Institute of Industrial and Control Engineering (IOC), Universitat Politècnica de Catalunya (UPC), Barcelona, Spain (raul.suarez@upc.edu). This work was partially supported by the Spanish Government through the projects DPI2013-40882-P, DPI2014-57757-R and DPI2016-80077-R. N. García is supported by the Generalitat de Catalunya through the grant FI-DGR 2016. that mimic the human ones, is relevant here because the goal of this work is the use of similar concepts applied to the motion planning of an anthropomorphic dual-arm system. A certain degree of coordination between the arms is required in almost all everyday tasks [13], [14]. Whereas all the DOF of the arms may be independently moved, some synergies exist when a human being performs a task with both arms. Using these synergies to improve the dual-arm motion planning is the goal of this work. The basic idea was presented in [15], and it is extended here including an analysis of the synergies associated to different tasks and the proposal of a new likeness index to evaluate the similarity between two tasks based on the movements done by the arms of a human operator to solve them. This is a first attempt to define task families according to the used synergies and employ them to improve the corresponding motion planning.

The paper is organized as follows. Section II presents the problem statement and the contributions of this work. Section III describes the determination of dual-arm synergies and their use in motion planning. Section IV introduces the task likeness index, which is one of the contributions of this work. Sections V describes the experimentation related to the use of synergies, which is the base for the use of the likeness index introduced in Section VI. Finally, Section VII presents some conclusions and future work and an Appendix details several formal proofs.

\section{PRoblem STATEMENT AND MAIN CONTRIBUtions}

The problem to be solved is the motion planning of a dual-arm system looking for a reduction of the planning complexity while trying to mimic the movements that a human does to solve a given task. With this aim, we use the synergies that exist in the dual-arm movements when humans solve different tasks. These synergies are employed to reduce the complexity of the planning phase through a reduction of the dimension of the search space, adapting, when possible, the search space to the task to be solved. Besides, the synergies obtained from human demonstrations while solving different tasks are used to establish an index of the similarity between two tasks. This allows the determination of a task clustering or taxonomy that is useful to optimize the planning phase, allowing a further improvement of the planning process as well as an improvement of the planned motions.

The information obtained from the samples of the task executions are used in this work to define an index that indicates the likeness between two tasks, a larger likeness means that the movements to perform the tasks are more similar. This opens new ways to further improve the task planning process as well as the resulting plan itself. The key idea is to determine clusters of similar tasks that can be planned with the same set of synergies in an efficient way, while another set of synergies would be better for some other tasks. This paper presents an approach to advance in this direction. The key points are:

1) Definition of the likeness between two tasks based on the synergy information obtained from their executions by the human operator. This will allow to classify or cluster the tasks into subsets than can be solved with similar movements.

2) Use the concept of likeness between tasks to obtain better plans for tasks that were not previously demonstrated. This is done by looking for synergies from a demonstrated "similar" task and use them to improve the planning procedure.

Although it is not in the scope of this work, the likeness index could be also used to determine a complete task taxonomy according to the movements done to solve the tasks. 


\section{PRELIMINARIES}

\section{A. Determination of dual-arm synergies}

The synergies of the dual-arm system can be processed in two possible ways. On the one side, the information about the joints of the human arms can be captured and directly used to look for their synergies, this generates the real synergies of the human arms, but then they must be mapped to the robotic dual-arm system in a non-trivial way when the kinematic structures are not completely equivalent (see an example in [16]). On the other side, the human arm movements can be mapped first onto the robotic system using a predefined mapping (e.g. [17]), so that when the human moves the arms the movements are automatically mapped to the robotic system, and then the synergies can be determined from the resulting values of the mechanical joints. In this way the synergies are directly obtained for the used robotic system in correspondence with the movements done by the human operator. In this work we use the second option.

In order to obtain the dual-arm synergies, a Principal Component Analysis (PCA) [18] is run over the set of obtained dual-arm configurations $\boldsymbol{\theta}_{i}$. The PCA identifies the directions where the samples have larger dispersion and returns a new orthogonal basis of the dual-arm configuration space. Each vector of this new basis has length equal to the dispersion of the data along it and the vectors are sorted by length in decreasing order (i.e. the first vector indicates the direction with maximum dispersion of the samples). These vectors are called Principal Motion Directions (PMDs) and represent the arm synergies. In the rest of the paper synergies and PMDs are used with equivalent meaning. This simple linear approximation is enough to capture the subspace where the demonstrated motions lie. It has also been demonstrated to be useful and capable of being implemented by a real-time algorithm [19] or a drive mechanism [20]. However, nonlinear approaches to obtain synergies have been also proposed, such as the Gaussian process latent variable model [21] or the unsupervised kernel regression [22].

Different tasks are demonstrated by human operators and, for each task, the corresponding synergy basis is computed, i.e. the set of PMDs. Let:

- $m$ be the dimension of the configuration space.

- $\boldsymbol{\sigma}=\left[\sigma_{1}, \ldots, \sigma_{m}\right]$ be the vector of eigenvalues from the PCA ordered such that $\sigma_{j} \geq \sigma_{j+1}$.

- $U=\left[\boldsymbol{u}_{1}, \ldots, \boldsymbol{u}_{m}\right]$ be the matrix of eigenvectors from the PCA ordered as the corresponding eigenvalues $\sigma_{j}$ in $\boldsymbol{\sigma}$.

- $\boldsymbol{\mu}$ be the barycenter of the samples used in the PCA.

- $\Sigma=U D^{2} U^{\top}$ be the covariance matrix of the samples used in the PCA, where $D$ is the diagonal matrix with the values of $\sigma$ in the diagonal.

Then, each task can be characterized by its synergy basis defined as $S=\left(\boldsymbol{\mu},\left[\sigma_{1} \boldsymbol{u}_{1}, \ldots, \sigma_{m} \boldsymbol{u}_{m}\right]\right)$.

\section{B. Planning using synergies}

Once the dual-arm synergies have been obtained they can be used to reduce the dimension of the planning space, and therefore reduce the complexity of the planning procedure.

Let $B$ be the $m$-dimensional box in the configuration space that contains the $(100-\alpha) \%$ of the normal multivariate distribution of the samples used in the PCA to obtain the PMDs (typical value for $\alpha$ is $5 \%) . B$ is centered at $\boldsymbol{\mu}$, with each side $j \in[1, m]$ aligned with $\boldsymbol{u}_{j}$ and with length $2 \lambda \sigma_{j}$ where $\lambda=\sqrt{2}$ erf $-1(\sqrt[m]{1-\alpha})$. Also, let $q \leq m$ be the number of the first PMDs that makes the accumulated variance be above a confidence level of $(100-\beta) \%$. Then, the planning will be done in the $q$-dimensional subspace, called $P M D$ subspace $B_{q}$, spanned by the first $q$ PMDs and interior to $B$.

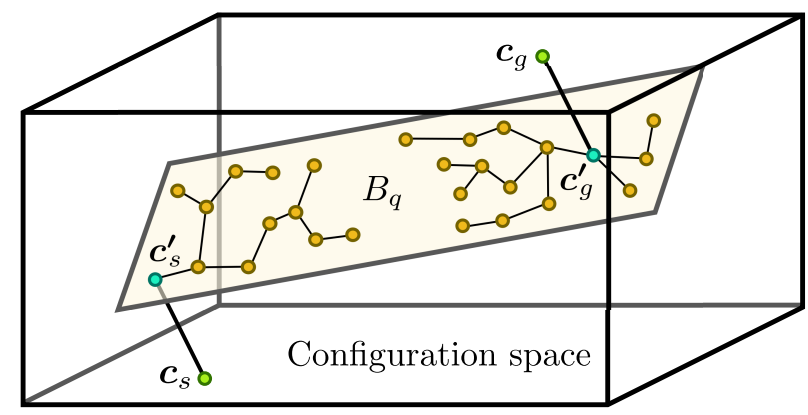

Figure 1. Conceptual representation of the whole task configuration space, and the PMD subspace $B_{q}$ where the planner works with the start and goal configurations $\left(\boldsymbol{c}_{s}\right.$ and $\boldsymbol{c}_{g}$ ) and their closest configurations in $B_{q}\left(\boldsymbol{c}_{s}^{\prime}\right.$ and $\left.\boldsymbol{c}_{g}^{\prime}\right)$.

Now, let $\boldsymbol{c}_{s} \in \mathcal{C}_{s}$ and $\boldsymbol{c}_{g} \in \mathcal{C}_{g}$ be respectively a start and a goal configuration of a task to be carried out by the dual-arm system, where $\mathcal{C}_{s}$ and $\mathcal{C}_{g}$ are the sets of collision-free configurations satisfying the constraints affecting the poses of the objects grasped by the hands at the initial and final states of the task, respectively. Now let $\boldsymbol{c}=\left\{\boldsymbol{c}_{s}, \boldsymbol{c}_{g}\right\}$ be a motion planning query, composed of a start and a goal configuration.

Then, once a manipulation task is defined, a large enough set of $N_{c}$ queries is selected from $\mathcal{C}_{s}$ and $\mathcal{C}_{g}$, satisfying the following conditions (see Fig. 1):

a) $\boldsymbol{c}$ is near the PMD subspace $B_{q}$ used to solve the task, i.e. the distances from $\boldsymbol{c}_{s}$ and $\boldsymbol{c}_{g}$ to the corresponding closest configurations in $B_{q}, \boldsymbol{c}_{s}^{\prime}$ and $\boldsymbol{c}_{g}^{\prime}$ respectively, are below a given threshold.

b) The configurations $\boldsymbol{c}_{s}^{\prime}$ and $\boldsymbol{c}_{g}^{\prime}$ as well as the rectilinear paths in the configuration space connecting $\boldsymbol{c}_{s}$ with $\boldsymbol{c}_{s}^{\prime}$ and $\boldsymbol{c}_{g}$ with $\boldsymbol{c}_{g}^{\prime}$ are collision-free.

Finally, a reduced set of $n_{c}$ samples is selected from the $N_{c}$ sampled queries. $n_{c}$ is an arbitrarily predefined number selected according to the available computational capacity, and the selection is done such that the selected samples are the closest ones to $B_{q}$. Then, for each of the selected $n_{c}$ queries, an instance of a RRT-Connect planner [4] is run in the PMD subspace to find a path between the configurations $\boldsymbol{c}_{s}^{\prime}$ and $\boldsymbol{c}_{g}^{\prime}$ corresponding to the query configurations $\boldsymbol{c}_{s}$ and $\boldsymbol{c}_{g}$, respectively, as illustrated in Fig. 1. All the planner instances run in parallel and once a solution path is found by one of them, the motion planning is stopped and all the other threads are killed.

Planning in $B_{q}$ is more efficient than doing it in the whole configuration space, because on the one hand it is done in a lower dimensional space and, on the other hand, less self-collision occur (since a high percentage of the samples mapped from the human motions lies there) [15].

\section{TASK LIKENESS}

In this section we propose an index of the likeness between two tasks using the synergy basis of each of them, i.e. the likeness between two tasks $A$ and $B$ is defined as the likeness $\mathcal{L}\left(S_{A}, S_{B}\right)$ between the corresponding synergy bases $S_{A}$ and $S_{B}$.

Let $\mathcal{N}_{A}$ and $\mathcal{N}_{B}$ be the multivariate normal distributions represented by each synergy basis $S_{A}$ and $S_{B}$, respectively, i.e. for each task $\Gamma \in\{A, B\}$

$$
\mathcal{N}_{\Gamma}=\mathcal{N}\left(\boldsymbol{\mu}_{\Gamma}, \Sigma_{\Gamma}\right)=(2 \pi)^{-\frac{m}{2}}\left|\Sigma_{\Gamma}\right|^{-\frac{1}{2}} e^{-\frac{1}{2}\left(\boldsymbol{x}-\boldsymbol{\mu}_{\Gamma}\right)^{\boldsymbol{\top}} \Sigma_{\Gamma}^{-1}\left(\boldsymbol{x}-\boldsymbol{\mu}_{\Gamma}\right)}
$$

Then, the likeness index $\mathcal{L}\left(S_{A}, S_{B}\right)$ is defined as

$$
\mathcal{L}\left(S_{A}, S_{B}\right)=\frac{\Phi_{A B}}{\Phi_{A B_{\max }}}
$$




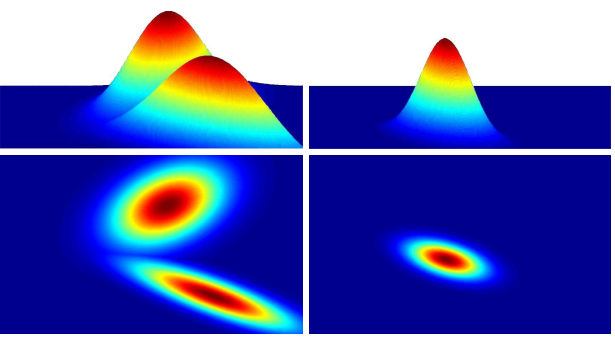

Figure 2. Surface plots of two bivariate normal distributions $\mathcal{N}_{A}$ and $\mathcal{N}_{B}$ (left), and their product (right), in both cases the top row is a perspective view and the bottom row is a top view. The units of the axes are not specified since they are irrelevant for this illustrative example.

where $\Phi_{A B}$ is a measure of the overlap between $\mathcal{N}_{A}$ and $\mathcal{N}_{B}$ all over the entire $m$-dimensional configuration space, and $\Phi_{A B_{\max }}$ is the upper bound value of $\Phi_{A B}$. Then, $\mathcal{L}\left(S_{A}, S_{B}\right) \in[0,1]$ with $\mathcal{L}\left(S_{A}, S_{B}\right)=1$ representing the maximum likeness, i.e. the tasks $A$ and $B$ could actually be the same task.

$\Phi_{A B}$ is defined as the integral of the product of $\mathcal{N}_{A}$ and $\mathcal{N}_{B}$ over the entire space

$$
\Phi_{A B}=\int_{-\infty}^{\infty} \cdots \int_{-\infty}^{\infty} \mathcal{N}_{A} \mathcal{N}_{B} d \boldsymbol{x}
$$

In practice, $\Phi_{A B}$ can be computed as (see the Appendix for the proof)

$$
\Phi_{A B}=\frac{e^{-\frac{1}{2}\left(\boldsymbol{\mu}_{A}-\boldsymbol{\mu}_{B}\right)^{\top}\left(\Sigma_{A}+\Sigma_{B}\right)^{-1}\left(\boldsymbol{\mu}_{A}-\boldsymbol{\mu}_{B}\right)}}{\sqrt{(2 \pi)^{m}\left|\Sigma_{A}+\Sigma_{B}\right|}}
$$

with the components $\left(\mu_{A_{j}}-\mu_{B_{j}}\right)$ expressed in the range $[-\pi, \pi)$. Note that since $\mu_{A_{j}}$ and $\mu_{B_{j}}$ are in the range $[-\pi, \pi)$, the simple signed difference angle between them could lie outside this range.

$\Phi_{A B}$ has the following bounds (see the Appendix for the proof)

$$
0<\Phi_{A B} \leq \Phi_{A B_{\max }}=\left(\pi^{\frac{m}{2}} \prod_{j=1}^{m}\left(\sigma_{A_{j}}+\sigma_{B_{j}}\right)\right)^{-1}
$$

with $\sigma_{A_{j}}$ and $\sigma_{B_{j}}$ being the $j$-th eigenvalue of the synergies $S_{A}$ and $S_{B}$ respectively.

Fig. 2 shows an example of two bivariate normal distributions $\mathcal{N}_{A}$ and $\mathcal{N}_{B}$ and the result of their product $\mathcal{N}_{A} \mathcal{N}_{B}$. The volume beneath the surface defined by $\mathcal{N}_{A} \mathcal{N}_{B}$ is the value of $\Phi_{A B}$. It is worth remarking that the product $\mathcal{N}_{A} \mathcal{N}_{B}$ is a normal distribution multiplied by a scale factor (see the Appendix for the proof).

\section{Planning USING SYNERgIES: IMPLEMENTATION AND DISCUSSION}

\section{A. Experimental setup}

The main elements used in the experimentation are (Fig. 3):

- A robotic dual-arm system composed of two industrial robot arms UR5 from Universal Robots with 6 DOF each one, which are assembled emulating the human arm configuration. Each robot arm is equipped with an Allegro Hand from Simlab with $16 \mathrm{DOF}$ (three fingers and a thumb with four independent joints each one).

- Two sensorized gloves CyberGlove, used to capture the orientations of the human operator hands. Each glove provides 22 joint-angle measurements: three flexion sensors per finger, four abduction sensors between the fingers, a palm-arch sensor and two sensors to measure the flexion and abduction of the wrist. These two last sensors are the only ones used in this work.

- Two magnetic wrist trackers Fastrak from Polhemus, used to capture the 6 DOF position and orientation of each user wrist referenced to the global frame.

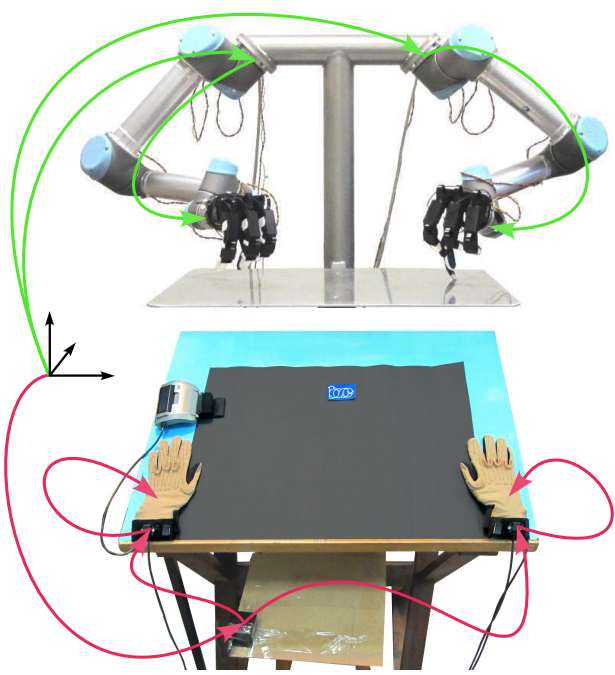

Figure 3. Dual-Arm system (top) and measurement equipment used to capture the movements of the human operator (bottom). The used transformations related to the robot, the gloves and the trackers are represented with arrows.

- A simulation tool, called The Kautham Project [23], with capabilities for collision detection, motion planning and graphical visualization of the whole system.

\section{B. Demonstration tasks}

The three following tasks were selected to illustrate the proposed approach (Fig. 4):

- An Assembly task in which the human operator must grasp a cylindrical box and a soda can, and then move them to a preassembly pose that allows the insertion of the can into the box.

- A Pouring task in which the human operator must grasp a glass and a soda can, and then pour the drink into the grasped glass.

- A Box task in which the human operator must grasp a cube and open a box, and then introduce the cube into the box.

Besides, looking for a general and practical application of the approach, we also capture the movements and obtain the PMDs when the operator freely moves both arms and hands in an unconstrained way (i.e. without performing any specific task) trying to cover the whole natural workspace in front of the body, we will refer to this as a Free-movement task. The task is finished when the operator considers that (s)he has covered the whole workspace. There is no guarantee that the operator actually covers the whole workspace, but it is expected that (s)he performs his most natural and evident movements.

Each of these four tasks was executed 10 times by 3 human operators using the arms without moving the rest of the body, generating representative sets of more than 10,000 samples per task. Pictures of the task goal were shown to the operators to instruct them. The initial positions of the objects were randomly located in given areas of the table, and the final goal positions were those where the operator comfortably execute the task in a natural way. This gives variability to the data while preserving the essence of the task motions.

\section{Task synergies}

In order to capture the movements of the operator arms, all the sensors are synchronized to take samples at the same time with a rate of $50 \mathrm{~Hz}$. Each sample contains a translation vector and a rotation quaternion read from each Fastrak tracker, 22 measurements 
a)
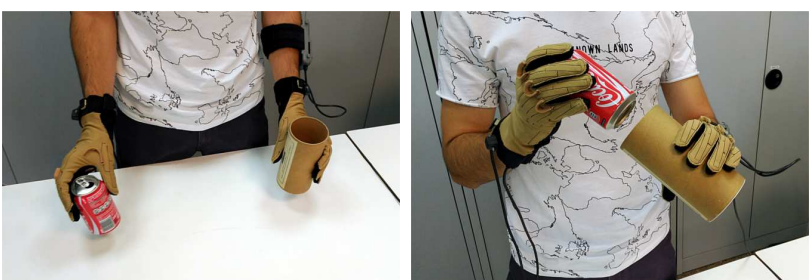

b)
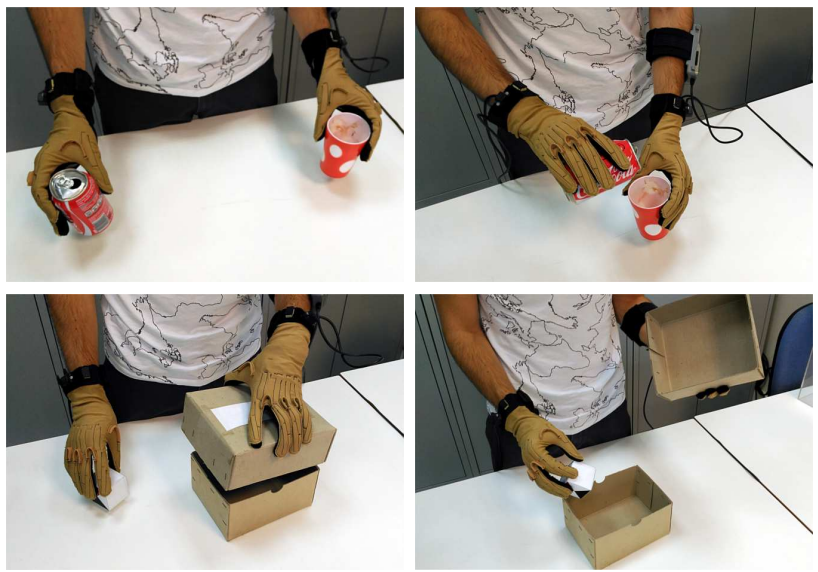

Figure 4. Human operator performing the demonstrations tasks while wearing the measurement equipment: a) Assembly task, b) Pouring task, c) Box task. The left and right columns show, respectively, examples of the start and goal configurations.

describing the positions of the finger joints and the hand orientation (flexion and abduction) read from each glove, a sample identification number, and the time in which it was captured. Nevertheless, from the information provided by the gloves only the values describing each hand orientation are actually used in this work. Therefore, each sample $s_{i}$ obtained from the demonstrations has 16 independent measurements, six for the pose of each wrist and two for the orientation of each hand.

Once the samples $s_{i}$ from the human movements have been captured, they are mapped to the robotic system. This mapping depends on the kinematic structure and particularities of the used robotic system. In this work, we simply consider the samples as being in the workspace of the dual-arm system, and then obtain the position of the arm joints by solving the inverse kinematics of the arms for each end effector configuration defined by each sample $s_{i}$. In this way, each sample $s_{i}$ generates a 12-dimensional configuration $\boldsymbol{\theta}_{i}$ of the dual-arm system. Note that redundant robotic arms may allow infinite solutions to the inverse kinematics problem, therefore some anthropomorphism criterion could be optimized while solving it (e.g. controlling the position of the robot elbows [24]).

Applying the process described in Section III, a 12-dimensional synergy basis $S_{A}$ was obtained for each task. Table I shows the resulting variances along each PMD for each task, which are graphically represented in Fig. 5. Note that for the Pouring task almost the $90 \%$ of the sample variance is associated with the first PMD, the second PMD has still some (low) relevance, but the other PMDs have a very small dispersion. This means that the task executions were quite repetitive, and that the task could (almost) be done considering only the first PMD (which implies the coordinated movement of several or all the system joints, but since this is done in a fixed coordinated way it is equivalent to a single degree of freedom). In the Assembly task there are two PMDs with non-negligible variance while in the Box task there are three PMDs with non-negligible variance. Regarding the Free-movement task, it can be seen that the first two PMDs concentrate the main sample variance although the
Table I

SAMPLE VARIANCE AND ACCUMULATED SAMPLE VARIANCE ALONG THE $j$-TH PMD AND TOTAL SAMPLE VARIANCE FOR EACH TASK.

\begin{tabular}{|c||c:c|c|c|c|c|c|c|}
\hline \multirow{2}{*}{$\begin{array}{c}j \text {-th } \\
\text { PMD }\end{array}$} & Assembly & \multicolumn{2}{c|}{ Pouring } & \multicolumn{2}{c|}{ Box } & \multicolumn{2}{c|}{ Free-mov. } \\
\cline { 2 - 8 } & var. & acc. & var. & acc. & var. & acc. & var. & acc. \\
\hline \hline 1 & $82.6 \%$ & $82.6 \%$ & $89.6 \%$ & $89.6 \%$ & $64.4 \%$ & $64.4 \%$ & $41.2 \%$ & $41.2 \%$ \\
\hline 2 & $16.3 \%$ & $98.9 \%$ & $5.7 \%$ & $95.3 \%$ & $22.7 \%$ & $87.1 \%$ & $32.2 \%$ & $73.3 \%$ \\
\hline 3 & $0.8 \%$ & $99.7 \%$ & $2.1 \%$ & $97.4 \%$ & $11.1 \%$ & $98.2 \%$ & $7.5 \%$ & $80.8 \%$ \\
\hline 4 & $0.1 \%$ & $99.8 \%$ & $1.8 \%$ & $99.2 \%$ & $1.0 \%$ & $99.2 \%$ & $5.1 \%$ & $85.9 \%$ \\
\hline 5 & $0.1 \%$ & $99.9 \%$ & $0.3 \%$ & $99.5 \%$ & $0.5 \%$ & $99.7 \%$ & $4.2 \%$ & $90.1 \%$ \\
\hline 6 & $0.0 \%$ & $99.9 \%$ & $0.2 \%$ & $99.7 \%$ & $0.2 \%$ & $99.9 \%$ & $3.3 \%$ & $93.4 \%$ \\
\hline 7 & $0.0 \%$ & $100 \%$ & $0.1 \%$ & $99.9 \%$ & $0.0 \%$ & $99.9 \%$ & $2.0 \%$ & $95.4 \%$ \\
\hline 8 & $0.0 \%$ & $100 \%$ & $0.1 \%$ & $99.9 \%$ & $0.0 \%$ & $100 \%$ & $1.6 \%$ & $97.0 \%$ \\
\hline 9 & $0.0 \%$ & $100 \%$ & $0.0 \%$ & $100 \%$ & $0.0 \%$ & $100 \%$ & $1.3 \%$ & $98.3 \%$ \\
\hline 10 & $0.0 \%$ & $100 \%$ & $0.0 \%$ & $100 \%$ & $0.0 \%$ & $100 \%$ & $0.9 \%$ & $99.2 \%$ \\
\hline 11 & $0.0 \%$ & $100 \%$ & $0.0 \%$ & $100 \%$ & $0.0 \%$ & $100 \%$ & $0.4 \%$ & $99.7 \%$ \\
\hline 12 & $0.0 \%$ & $100 \%$ & $0.0 \%$ & $100 \%$ & $0.0 \%$ & $100 \%$ & $0.3 \%$ & $100 \%$ \\
\hline \hline Total & $7.942 \mathrm{rad}^{2}$ & $7.569 \mathrm{rad}^{2}$ & $6.660 \mathrm{rad}^{2}$ & $9.994 \mathrm{rad}^{2}$ \\
\hline
\end{tabular}

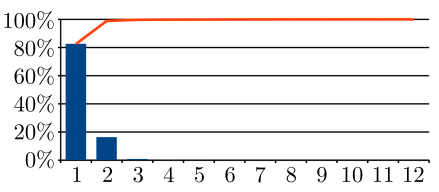

(a) Assembly

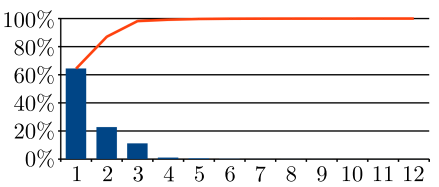

(c) Box

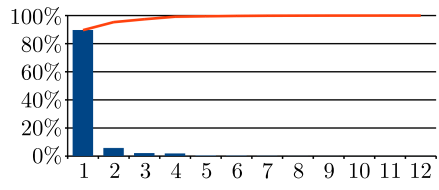

(b) Pouring

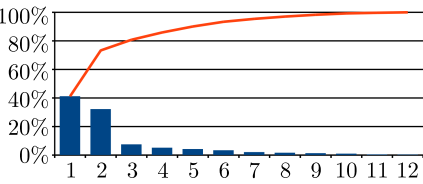

(d) Free-movement
Figure 5. Accumulated sample variance versus the number of PMDs.

dispersion is still relevant along the first six or seven PMDs. This is an expected effect since the operator has more freedom to perform the movements, which can also be seen in the total variance of the samples, clearly greater than in the other tasks (see Table I).

\section{Effect of using PMDs in motion planning}

For each task the planning procedure was run using:

1) The whole 12-dimensional dual-arm configuration space.

2) The $q$-dimensional subspace $B_{q}$ of the planned task.

3) The $q$-dimensional subspace $B_{q}$ of the other tasks.

Note that while the full dimension of the problem is always $m=12$ (the total number of DOF of the two arms), the reduced dimension $q$ has different values depending on the executed task, we selected $\beta=5$ in order to cover $95 \%$ of the the accumulated variance of the samples (see Subsection III-B), and therefore results $q=2$ for the Assembly and Pouring tasks, $q=3$ for the Box task and $q=7$ for the Free-movement task. For each task we choose to use $n_{c}=10$ different task queries selected from an initial set of $N_{c}=100$ (see Section III).

For the Assembly task, the set of goals $N_{c}$ was generated satisfying the geometrical constraints necessary for the assembly of the soda can into a cylindrical box (see Fig. 4a). This results in a 7-dimensional goal space, i.e. the box can be in different positions and orientations (six DOF) and the can is allowed to rotate around its axis while satisfying the pre-assembly pose constraints (one additional DOF).

For the Pouring task the goal space is also 7-dimensional, the glass must be vertical and resting on the table (three DOF) and the can must 
Table II

AVERAGE MOTION-PLANNING RESULTS WHEN THE TASKS ARE SOLVED WITHOUT PMDS, WITH TASK-SPECIFIC PMDS AND WITH OTHER TASKS PMDS.

\begin{tabular}{|l||c|c|c|c|c|c|c|c|c|c|c|c|c|c|c|c|}
\hline Task & \multicolumn{9}{|c|}{ Assembly } & \multicolumn{4}{c|}{ Pouring } & \multicolumn{4}{c|}{ Box } \\
\hline Solved with PMDs of: & - & Ass. & Pour. & Box & Free. & - & Ass. & Pour. & Box & Free. & - & Ass. & Pour. & Box & Free. \\
\hline \hline \# of used PMDs & 0 & 2 & 2 & 3 & 7 & 0 & 2 & 2 & 3 & 7 & 0 & 2 & 2 & 3 & 7 \\
\hline Space dimension & 12 & 2 & 2 & 3 & 7 & 12 & 2 & 2 & 3 & 7 & 12 & 2 & 2 & 3 & 7 \\
\hline Success rate [\%] & 100 & 100 & 100 & 100 & 100 & 100 & 100 & 100 & 100 & 100 & 100 & 100 & 100 & 100 & 100 \\
\hline Used time [ms] & 82.93 & 42.05 & 62.09 & 73.42 & 66.54 & 127.31 & 84.66 & 40.99 & 65.25 & 103.00 & 143.14 & 93.88 & 83.23 & 46.22 & 103.36 \\
\hline \# of tree nodes & 6.27 & 4.02 & 4.49 & 5.04 & 4.95 & 8.50 & 6.12 & 4.00 & 4.85 & 6.93 & 11.70 & 8.05 & 6.39 & 4.04 & 8.84 \\
\hline Solution length [rad]* & 3.761 & 2.112 & 2.343 & 2.954 & 2.691 & 5.835 & 4.012 & 2.240 & 2.608 & 4.859 & 9.186 & 5.868 & 4.773 & 3.603 & 6.624 \\
\hline Valid segments [\%] & 80.34 & 100 & 100 & 100 & 97.77 & 84.64 & 100 & 100 & 100 & 100 & 81.75 & 95.80 & 100 & 100 & 98.78 \\
\hline \# of collision checks & 65.28 & 22.01 & 35.02 & 45.08 & 37.71 & 161.76 & 61.43 & 19.17 & 37.21 & 72.92 & 212.76 & 85.16 & 64.61 & 30.49 & 123.66 \\
\hline Path in $B_{q}[\%]^{* *}$ & 0 & 89.91 & 89.83 & 90.09 & 90.62 & 0 & 89.49 & 87.43 & 85.53 & 92.07 & 0 & 93.83 & 81.11 & 95.57 & 94.93 \\
\hline
\end{tabular}

* Evaluated as the summation of the joints movements in radians

** Percentage of the whole path contained in $B_{q}$, i.e. without considering the segments from $\boldsymbol{c}_{s}$ to $\boldsymbol{c}_{s}^{\prime}$ and from $\boldsymbol{c}_{g}^{\prime}$ to $\boldsymbol{c}_{g}$ (see Fig. 1).

have the opening exactly above within a predefined height range (one additional DOF) and with any orientation (three additional DOF). Note that the rotation about the can axis impose constraints on the can grasping, and the proper rotation about an horizontal axis depends on the quantity of liquid in the can, but for illustrative purposes in this work we simply considered this degree of freedom by imposing a small predefined rotation range (see Fig. 4b).

For the Box task the goal space is 6-dimensional, the box can be in any position and orientation on the table (three DOF) and the cube must be placed at a predefined position inside the box with any orientation (three additional DOF). It is assumed that the left and right hands are already grasping the box cover and the cube respectively at the start configuration (see Fig. 4c) Therefore, the start configuration depends on the position of the box for the left hand while for the right hand it is fixed. Similarly, the goal configuration depends on the position of the box for the right hand while for the left hand it is fixed.

For each task, an instance of the planner was run in parallel for each goal configuration, stopping the motion planner when a valid solution path was found by one of the instances. If the planner could not solve the task within a predefined time restriction of 100 seconds the run was considered as a failure. The Open Motion Planning Library (OMPL [25]) implementation of the RRT-Connect has been used encapsulated within The Kautham Project (the used planning and simulation environment [23]).

Table II shows the average planning results obtained after 100 executions for each case of each task, running in a $3.40-\mathrm{GHz}$ Intel i7-3770, 8-GB RAM PC.

The experimental results show that comparing with the planning in the whole configuration space (i.e. without the use of synergies):

- The use of PMDs increases significantly the probability of obtaining collision-free configurations (fewer self-collisions occur), thus reducing the computational time.

- The use of PMDs allows a reduction of the dimension of the search space, which reduces also the number of nodes and edges of the tree and hence reduces the memory requirements.

It must be highlighted that these aspects are more pronounced when task-specific PMDs are used, but, for general applications, the utility of the PMDs obtained with the Free-movement task is still relevant, since they also improve the results compared with planning without using PMDs.
Table III

LiKENESS $\mathcal{L}\left(S_{A}, S_{B}\right)$ BETWEEN THE CONSIDERED TASKS.

\begin{tabular}{|c||c|c|c|c|}
\hline Tasks & Assembly & Pouring & Box & Free-mov. \\
\hline \hline Assembly & 1 & 0.1081 & 0.0114 & 0.6104 \\
\hline Pouring & 0.1081 & 1 & 0.0035 & 0.5699 \\
\hline Box & 0.0114 & 0.0035 & 1 & 0.6829 \\
\hline Free-mov. & 0.6104 & 0.5699 & 0.6829 & 1 \\
\hline
\end{tabular}

\section{Planning USing the TASK LiKeness: IMPLEMENTATION AND DISCUSSION}

\section{A. Relation between task-dependent synergies and task likeness}

Table III shows the likeness $\mathcal{L}\left(S_{A}, S_{B}\right)$ between the demonstration tasks obtained with the procedure presented in Section IV. It can be seen that the Free-movement task is more similar to all the other tasks, while these tasks are more dissimilar between them.

In order to provide a graphical representation of the likeness between the tasks, we define a proximity index $\mathcal{D}\left(S_{A}, S_{B}\right)$ as

$$
\mathcal{D}\left(S_{A}, S_{B}\right)=1-\mathcal{L}\left(S_{A}, S_{B}\right) \in[0,1]
$$

Even when $\mathcal{D}$ is not a real distance, since it does not satisfy the triangle inequality (i.e. the distance between two points must be the shortest distance along any path between them), it is still possible to represent the synergy bases of the four considered tasks as points in a 3D Euclidean space such that the Euclidean distances $d\left(S_{i}, S_{j}\right)$ between these points minimize the maximum relative error with respect to the corresponding proximity indices $\mathcal{D}$, i.e minimizing

$$
\max _{i \neq j}\left(\frac{d\left(S_{i}, S_{j}\right)-\mathcal{D}\left(S_{i}, S_{j}\right)}{\mathcal{D}\left(S_{i}, S_{j}\right)}\right)
$$

By doing this, it results that the four points are approximately coplanar (the Free-movement task is at 0.0001 distance units far from the plane defined by the other three tasks, while the distance between any two tasks is higher by about three orders of magnitude), thus the synergies can actually be represented in a 2D Euclidean space, as shown in Fig. 6. This representation gives a clear intuitive view of the relation between the tasks by approximating the likeness $\mathcal{L}$ with the distances between them. Note that the Free-movement task lies inside the triangle defined by the other tasks. This confirms the idea of using this synergy basis for general applications.

The likeness $\mathcal{L}$ can be used to classify the tasks into families or clusters, and it is expected that solving a task with the PMDs of a more alike task would result in a faster and better motion planning. 


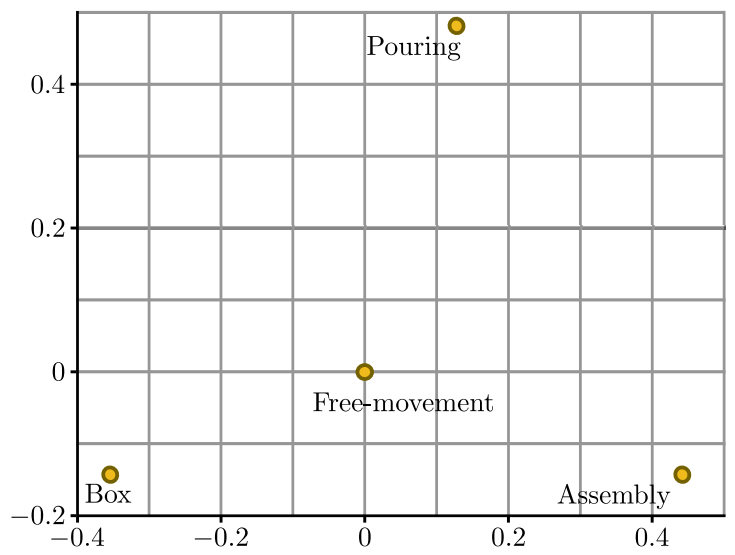

Figure 6. Representation of the demonstrated tasks in a 2-dimensional space based on the $\mathcal{L}$ values between the tasks. The origin has been set in the Free-movement task and the axes orientation was arbitrarily selected. The distance between two tasks is, by definition, constrained to the $[0,1]$ interval, therefore the axes are unit-less.

In order to test the real usefulness of $\mathcal{L}$, we checked whether there is a relation between $t_{A_{B}}$, the average time employed to solve the motion planning of task $A$ using the PMDs of task $B$ (see Table II), and $\mathcal{L}\left(S_{A}, S_{B}\right)$, the likeness between tasks $A$ and $B$ (see Table III). Since the tasks have different degrees of difficulty they inherently require different times $t$, thus the $t$ values were normalized to $\hat{t} \in[0,1]$ using the minimum time $t_{A_{\min }}$ and the maximum time $t_{A_{\max }}$ needed to solve a task $A$. Then, the values $\hat{t}_{A_{B}}$ were computed as:

$$
\hat{t}_{A_{B}}=\frac{t_{A_{B}}-t_{A_{\min }}}{t_{A_{\max }}-t_{A_{\min }}}
$$

It must be highlighted that for all the tasks, the minimum and maximum times are obtained respectively when the task-specific PMDs are used and when no PMDs are used. Furthermore, since the dimension of the search space plays a very important role, the number $q$ of PMDs used in the motion planning is also considered in the study of $\hat{t}$, i.e. $\hat{t}=\hat{t}(\mathcal{L}, q)$.

Using the data in Tables II and III, Fig. 7 shows the normalized time $\hat{t}$ as a function of the likeness $\mathcal{L}$ and the space dimension $q$ for the three tasks solved with their task-specific PMDs, the PMDs of the other two tasks and the PMDs of the Free-movement task. A plane $\hat{t}=\kappa_{0}+\kappa_{\mathcal{L}} \mathcal{L}+\kappa_{q} q$ has been fitted to the values of $\hat{t}$ as a first-order approximation. As it was expected the coefficient $\kappa_{\mathcal{L}}$ is negative, i.e. $\hat{t}$ decreases with $\mathcal{L}$. In addition, $\kappa_{q}$ is positive. Similar results are obtained when, instead of using the average time $\hat{t}$, the average path length or the average number of collisions are plotted. This verifies the hypothesis that using the PMDs of an alike task according to $\mathcal{L}$ produces better motion planning results (i.e. less planning time and shorter paths) for the same value of $q$.

\section{B. Motion planning using task likeness}

Consider that there is a new task to be solved but it has not been previously demonstrated, so a task-specific synergy basis is not available for it. The procedure proposed to improve the motion planning for this new task is:

1) Use of the PMDs of the Free-movement task to obtain a first plan that solves this non-demonstrated task.

2) Run a PCA using the samples of this first plan to obtain a new set of PMDs, i.e. obtaining a synergy basis $\hat{S}_{V}$ from only one virtual execution.

3) Search for the synergy basis $S_{A}$ most alike to $\hat{S}_{V}$. This is done by looking for the basis $S_{A}$ that maximizes $\mathcal{L}\left(\hat{S}_{V}, S_{A}\right)$ among all the demonstrated tasks.

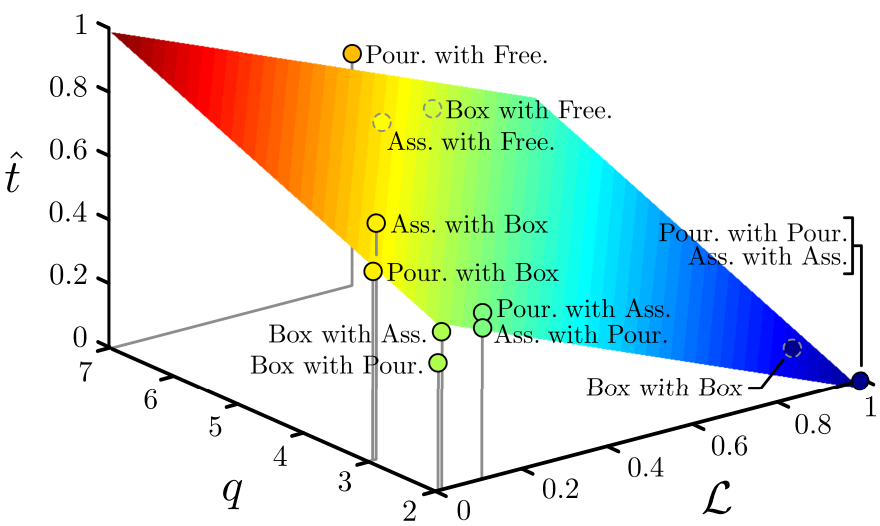

Figure 7. Normalized time $\hat{t} \in[0,1]$ as a function of the likeness $\mathcal{L} \in[0,1]$ and the number $q$ of PMDs used, for the considered tasks solved with the own PMDs and the ones of the other tasks. It is also shown the fitted plane $\hat{t}=\kappa_{0}+\kappa_{\mathcal{L}} \mathcal{L}+\kappa_{q} q$.

Table IV

LIKENESS $\mathcal{L}\left(\hat{S}_{V}, S_{A}\right)$ BETWEEN THE VIRTUAL AND THE DEMONSTRATED SYNERGY BASES*.

\begin{tabular}{|c||c|c|c:c|}
\hline Tasks & Assembly & Pouring & Box & Free-mov. \\
\hline \hline Virtual Assembly & $\mathbf{0 . 2 4 1 6}$ & 0.1916 & 0.1021 & 0.4966 \\
\hline Virtual Pouring & 0.2079 & $\mathbf{0 . 3 4 4 8}$ & 0.0645 & 0.4739 \\
\hline Virtual Box & 0.0999 & 0.1005 & $\mathbf{0 . 2 1 4 6}$ & 0.5132 \\
\hdashline Virtual Bottle & $\mathbf{0 . 4 0 2 0}$ & 0.1082 & 0.1254 & 0.6559 \\
\hline
\end{tabular}

* The likeness values between each virtual synergy basis and its closest demonstrated synergy basis are marked in bold, regardless of the basis of the Free-movement task (i.e. the synergy basis with which the virtual synergy basis was obtained).

4) Use of $S_{A}$ in a new motion planning process.

In order to check the validity of this procedure, a PCA was run on the samples obtained from one motion plan of each task generated using the Free-movement PMDs. This generates a new synergy basis for each task, and the likeness between this basis and the synergy bases obtained from the human demonstrations is given in Table IV. In all the cases the most alike synergy basis is the task-specific one, e.g. the likeness index between the virtual Assembly and the Assembly tasks is higher than the likeness index between the virtual Assembly and the other tasks, disregarding the Free-movement task, that was used to generate the virtual synergies.

The experimental results show that this approach improves the planning process. Nevertheless, since there are random searches in the whole process, the improvement can not be always ensured.

For illustrative purposes, this procedure was also used to solve the motion planning of a new, non-demonstrated task, called Bottle task. In this task, the dual-arm robot must grasp a half-full bottle with one hand and its cap with the other hand, and tap the bottle (see Fig. 8). Similarly to the Assembly task, the goal space is also 7-dimensional. However, in this case the goal pose of the assembled objects, i.e. the bottle tapped by the cap, is highly restricted in orientation to avoid pouring the liquid. Following the proposed procedure, a virtual synergy basis for the Bottle task was obtained using the Free-movement PMDs and the likeness indices with respect to the demonstrated synergy bases were computed. The Assembly task results to be the most alike task, as it was intuitively expected (see Table IV). Finally, the motion planning is solved again using the Assembly PMDs. The solutions obtained with this procedure results in movements of the robotic arms that have a natural appearance, even though the motion planning was solved using the synergies of 


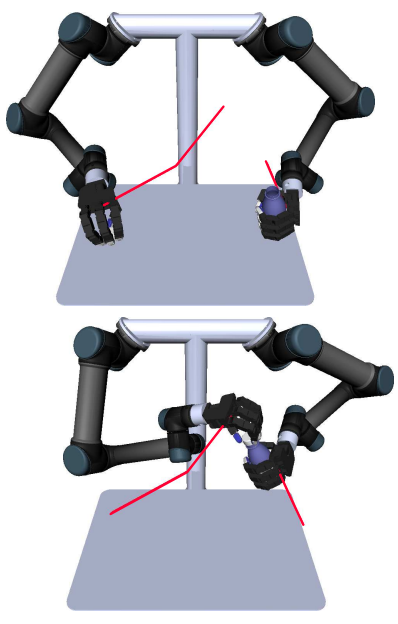

a)
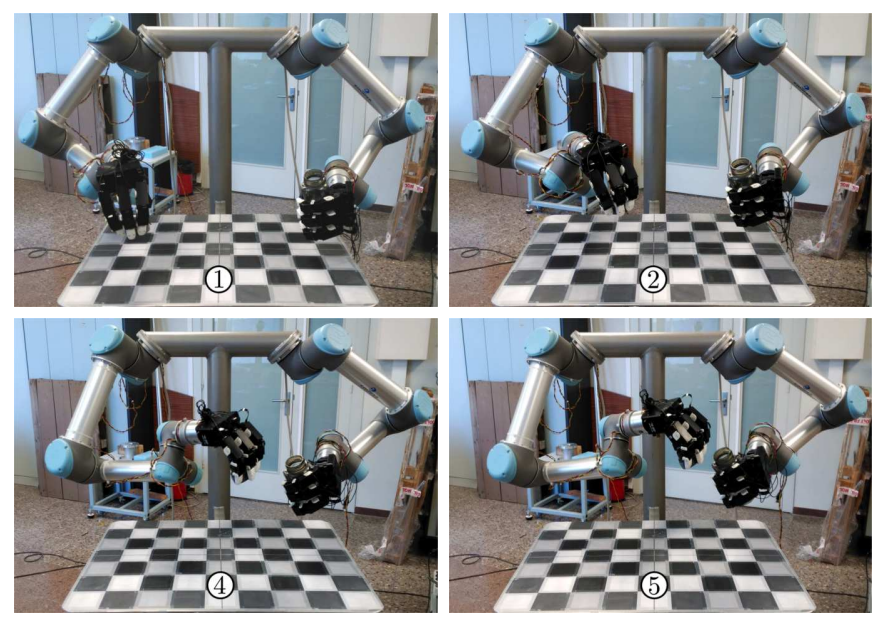

b)

Figure 8. Example of a solution path for the Bottle task obtained using the first two PMDs of the Assembly task (i.e. its closest task): a) start and goal configurations in the simulation environment, showing the planned paths; b) snapshots of the path execution with the real dual-arm system.

a different task. Snapshots of an instance of the obtained solution paths are shown in Fig. 8.

\section{DISCUSSION AND FUTURE WORK}

This paper has dealt with the determination of couplings, called synergies or PMDs, between the DOF of the arms of an operator, while performing different tasks, in order to use them to improve the motion planning process and the plan itself when a dual-arm robotic system performs the same or new tasks. The approach was detailed and implemented using an specific dual-arm system composed of two UR5 manipulators, although it can be mimicked for any other anthropomorphic dual-arm system.

The most relevant synergies define a lower-dimensional space where the motion planning can be done efficiently. The validity of the approach has been demonstrated analyzing the movements done to solve three different tasks plus a set of general movements freely done in the workspace (a Free-movement task). The results effectively showed that the use of the synergy space for the motion planning greatly reduces the computational time, basically due to the fact that the sampling procedure resulted in fewer self-collision configurations, and also to the planning in a reduced search space of lower dimension.

The paper has also proposed a likeness index between synergy bases, which indicates the similarity of two tasks according to the similarity of the movements done to solve them. The experimental results showed the relevance of this likeness index. After solving each task with different synergies it was demonstrated that using its task-specific synergies produces the best results, and in the other cases the higher the likeness between the used synergies and the task-specific ones the better the results, as long as the same number of PMDs is used. Moreover, a procedure to tackle the motion planning for new tasks has been also introduced, i.e. for tasks that have not been previously demonstrated by a user and therefore no task-specific synergy bases were available. A first clear result is that using the Free-movement synergies to plan a new task produces better results than not using synergies at all. A second option to further improve the results is the use of this first solution to compute a new (nondemonstrated) synergy basis, then, search the set of available synergy bases for the most likely one and, finally, use this existing synergy basis to recompute the solution path. As shown in the presented examples, the results were always improved.

The proposal opens several interesting potential research lines, such as the generation of a taxonomy of tasks based on the motions that humans do to solve them, the use of learning procedures to improve the set of synergies each time a new task is executed, the use of synergies in the space of the joint velocities of the arms in order to better mimic human movements during task executions, and the use of physics-based motion planners when interaction with the environment is allowed. Some works related to the last two research lines have been already developed [26], [27].

\section{APPENDIX}

This appendix describes the derivation of Eq. (4) and (5), and proves that the product of two multivariate normal distributions $\mathcal{N}_{A}$ and $\mathcal{N}_{B}$ is a multivariate normal distribution $\mathcal{N}_{C}$ except for a scale factor $\gamma$ (i.e. $\mathcal{N}_{A} \mathcal{N}_{B}=\gamma \mathcal{N}_{C}$ ).

Proof that $\mathcal{N}_{A} \mathcal{N}_{B}=\gamma \mathcal{N}_{C}$ : Using Eq. (1), $\mathcal{N}_{A} \mathcal{N}_{B}$ results

$$
\begin{aligned}
\mathcal{N}_{A} \mathcal{N}_{B} & =\frac{e^{-\frac{1}{2}\left(\boldsymbol{\mu}_{A}^{\top} \Sigma_{A}^{-1} \boldsymbol{\mu}_{A}+\boldsymbol{\mu}_{B}^{\top} \Sigma_{B}^{-1} \boldsymbol{\mu}_{B}+\boldsymbol{x}^{\top}\left(\Sigma_{A}^{-1}+\Sigma_{B}^{-1}\right) \boldsymbol{x}-2\left(\boldsymbol{\mu}_{A}^{\top} \Sigma_{A}^{-1}+\boldsymbol{\mu}_{B}^{\top} \Sigma_{B}^{-1}\right) \boldsymbol{x}\right)}}{(2 \pi)^{m} \sqrt{\left|\Sigma_{A} \| \Sigma_{B}\right|}} \\
& =\frac{e^{-\frac{1}{2}\left(\boldsymbol{\mu}_{A}^{\top} \Sigma_{A}^{-1} \boldsymbol{\mu}_{A}+\boldsymbol{\mu}_{B}^{\top} \Sigma_{B}^{-1} \boldsymbol{\mu}_{B}-\boldsymbol{\mu}_{C}^{\top} \Sigma_{C}^{-1} \boldsymbol{\mu}_{C}+\left(\boldsymbol{x}-\boldsymbol{\mu}_{C}\right)^{\top} \Sigma_{C}^{-1}\left(\boldsymbol{x}-\boldsymbol{\mu}_{C}\right)\right)}}{(2 \pi)^{m} \sqrt{\left|\Sigma_{A}\right| \Sigma_{B} \mid}} \\
& =\frac{e^{-\frac{1}{2}\left(\boldsymbol{\mu}_{A}^{\top} \Sigma_{A}^{-1} \boldsymbol{\mu}_{A}+\boldsymbol{\mu}_{B}^{\top} \Sigma_{B}^{-1} \boldsymbol{\mu}_{B}-\boldsymbol{\mu}_{C}^{\top} \Sigma_{C}^{-1} \boldsymbol{\mu}_{C}\right)}}{\sqrt{(2 \pi)^{m}\left|\Sigma_{A}\left\|\Sigma_{B}\right\| \Sigma_{C}\right|^{-1}}} \mathcal{N}_{C}=\gamma \mathcal{N}_{C}
\end{aligned}
$$

where $\Sigma_{C}=\left(\Sigma_{A}^{-1}+\Sigma_{B}^{-1}\right)^{-1}$, and $\boldsymbol{\mu}_{C}=\Sigma_{C}\left(\Sigma_{A}^{-1} \boldsymbol{\mu}_{A}+\Sigma_{B}^{-1} \boldsymbol{\mu}_{B}\right)$.

Proof of Eq. (4): On the first hand, from the matrix inversion lemma [28] the following equalities are derived

$$
\begin{gathered}
\Sigma_{C}=\left(\Sigma_{A}^{-1}+\Sigma_{B}^{-1}\right)^{-1}=\left\{\begin{array}{l}
\Sigma_{A}-\Sigma_{A}\left(\Sigma_{A}+\Sigma_{B}\right)^{-1} \Sigma_{A} \\
\Sigma_{B}-\Sigma_{B}\left(\Sigma_{A}+\Sigma_{B}\right)^{-1} \Sigma_{B}
\end{array}\right. \\
\begin{aligned}
\Sigma_{A}^{-1} \Sigma_{C} \Sigma_{B}^{-1} & =\Sigma_{A}^{-1}\left(\Sigma_{C}\left(\Sigma_{A}^{-1}+\Sigma_{B}^{-1}\right)-\Sigma_{C} \Sigma_{A}^{-1}\right)=\Sigma_{A}^{-1}\left(I-\Sigma_{C} \Sigma_{A}^{-1}\right) \\
& =\Sigma_{A}^{-1}-\Sigma_{A}^{-1} \Sigma_{C} \Sigma_{A}^{-1}=\left(\Sigma_{A}+\Sigma_{B}\right)^{-1}
\end{aligned}
\end{gathered}
$$

where $I$ is the identity matrix. Therefore,

$$
\begin{aligned}
& \boldsymbol{\mu}_{A}^{\top} \Sigma_{A}^{-1} \boldsymbol{\mu}_{A}+\boldsymbol{\mu}_{B}^{\top} \Sigma_{B}^{-1} \boldsymbol{\mu}_{B}-\boldsymbol{\mu}_{C}^{\top} \Sigma_{C}^{-1} \boldsymbol{\mu}_{C} \\
& =\boldsymbol{\mu}_{A}^{\top}\left(\Sigma_{A}+\Sigma_{B}\right) \overline{\boldsymbol{\mu}}_{A}^{-1}+\boldsymbol{\mu}_{B}^{\top}\left(\Sigma_{A}+\Sigma_{B}\right) \overline{\boldsymbol{\mu}}_{B}-2 \boldsymbol{\mu}_{A}^{\top}\left(\Sigma_{A}+\Sigma_{B}\right) \overline{\boldsymbol{\mu}}_{B}^{-1} \\
& =\left(\boldsymbol{\mu}_{A}-\boldsymbol{\mu}_{B}\right)^{\top}\left(\Sigma_{A}+\Sigma_{B}\right)^{-1}\left(\boldsymbol{\mu}_{A}-\boldsymbol{\mu}_{B}\right)
\end{aligned}
$$

On the other hand, we know by the matrix determinant lemma [29] that

$$
\left|\Sigma_{C}\right|=\left|\Sigma_{A}^{-1}+\Sigma_{B}^{-1}\right|^{-1}=\left|\Sigma_{A}\left\|\Sigma_{B}\right\| \Sigma_{A}+\Sigma_{B}\right|^{-1}
$$


These statements simplify Eq. (9) to

$$
\mathcal{N}_{A} \mathcal{N}_{B}=\frac{e^{-\frac{1}{2}\left(\mu_{A}-\mu_{B}\right)^{\top}\left(\Sigma_{A}+\Sigma_{B}\right)^{-1}\left(\boldsymbol{\mu}_{A^{-}} \boldsymbol{\mu}_{B}\right)}}{\sqrt{(2 \pi)^{m}\left|\Sigma_{A}+\Sigma_{B}\right|}} \mathcal{N}_{C}
$$

And since the integral of a probability density function over the entire space is 1 by definition, $\Phi_{A B}$ can be expressed as

$$
\Phi_{A B}=\int_{-\infty}^{\infty} \cdots \int_{-\infty}^{\infty} \mathcal{N}_{A} \mathcal{N}_{B} d \boldsymbol{x}=\frac{e^{-\frac{1}{2}\left(\boldsymbol{\mu}_{A}-\boldsymbol{\mu}_{B}\right)^{\top}\left(\Sigma_{A}+\Sigma_{B}\right)^{-1}\left(\boldsymbol{\mu}_{A}-\boldsymbol{\mu}_{B}\right)}}{\sqrt{(2 \pi)^{m}\left|\Sigma_{A}+\Sigma_{B}\right|}}
$$

Proof of Eq. (5): On the first hand, we know by the results in [30] that

$$
\left|\Sigma_{A}+\Sigma_{B}\right| \geq \prod_{j=1}^{m}\left(\sigma_{A_{j}}^{2}+\sigma_{B_{j}}^{2}\right)
$$

On the other hand, $e^{-x^{2}} \in(0,1], x \in \mathbb{R}$. With this in mind, we can have lower and upper bounds for $\Phi_{A B}$

$$
0<\Phi_{A B} \leq \widetilde{\Phi}_{A B_{\max }}=\left((2 \pi)^{m} \prod_{j=1}^{m}\left(\sigma_{A_{j}}^{2}+\sigma_{B_{j}}^{2}\right)\right)^{-\frac{1}{2}}
$$

Note that the equality is held when $\boldsymbol{\mu}_{A}=\boldsymbol{\mu}_{B}$ and $U_{A}=U_{B}$. Nevertheless, $\widetilde{\Phi}_{A B_{\max }}$ is not the upper bound we want since the inequality becomes an equality no matter what the eigenvalues of $\Sigma_{A}$ and $\Sigma_{B}$ are; $\mathcal{L}\left(S_{A}, S_{B}\right)$ is desired to be 1 (i.e. $\left.\Phi_{A B}=\Phi_{A B_{\max }}\right)$ if and only if $S_{A}$ and $S_{B}$ are exactly the same synergy basis (i.e. $\boldsymbol{\mu}_{A}=\boldsymbol{\mu}_{B}$ and $\left.\Sigma_{A}=\Sigma_{B}\right)$. However, using the fact that $2\left(x^{2}+y^{2}\right) \geq(|x|+|y|)^{2}$ (the equality holds $\forall x=y$ ), now we can define our global bounds as

$$
0<\Phi_{A B} \leq \widetilde{\Phi}_{A B_{\max }} \leq \Phi_{A B_{\max }}=\left(\pi^{\frac{m}{2}} \prod_{j=1}^{m}\left(\sigma_{A_{j}}+\sigma_{B_{j}}\right)\right)^{-1}
$$

\section{REFERENCES}

[1] J.-C. Latombe, Robot Motion Planning. Norwell, MA, USA: Kluwer Academic Publishers, 1991.

[2] M. Elbanhawi and M. Simic, "Sampling-based robot motion planning: A review," IEEE Access, vol. 2, pp. 56-77, Jan. 2014.

[3] L. E. Kavraki, P. Svestka, J. Latombe, and M. Overmars, "Probabilistic roadmaps for path planning in high-dimensional configuration spaces," in Proc. IEEE Int. Conf. Robotics and Automation, Aug. 1996, pp. 566580.

[4] J. J. Kuffner and S. LaValle, "RRT-Connect: An efficient approach to single-query path planning," in Proc. IEEE Int. Conf. Robotics and Automation, Apr. 2000, pp. 995-1001.

[5] L. Basañez and R. Suárez, "Teleoperation," in Springer Handbook of Automation, S. Nof, Ed. Springer-Verlag, 2009, pp. 449-468.

[6] M. Santello, M. Flanders, and J. F. Soechting, "Postural hand synergies for tool use," J. Neuroscience, vol. 18, no. 23, pp. 10 105-10 115, Dec. 1998.

[7] M. T. Ciocarlie and P. K. Allen, "Hand posture subspaces for dexterous robotic grasping," Int. J. Robotics Research, vol. 28, no. 7, pp. 851-867, July 2009.

[8] M. Gabiccini, A. Bicchi, D. Prattichizzo, and M. Malvezzi, "On the role of hand synergies in the optimal choice of grasping forces," Autonomous Robots, vol. 31, pp. 235-252, July 2011.

[9] D. Prattichizzo, M. Malvezzi, M. Gabiccini, and A. Bicchi, "On motion and force controllability of precision grasps with hands actuated by soft synergies," IEEE Trans. Robotics, vol. 29, no. 6, pp. 1440 - 1456, Dec. 2013.
[10] J. Rosell, R. Suárez, C. Rosales, and A. Pérez, "Autonomous motion planning of a hand-arm robotic system based on captured human-like hand postures," Autonomous Robots, vol. 31, no. 1, pp. 87-102, May 2011.

[11] J. Rosell and R. Suárez, "Using hand synergies as an optimality criterion for planning human-like motions for mechanical hands," in Proc. IEEERAS Int. Conf. Humanoid Robots, Nov. 2014.

[12] S. Sun, C. Rosales, and R. Suárez, "Study of coordinated motions of the human hand for robotic applications," in Proc. IEEE Int. Conf. Information and Automation, June 2010, pp. 776-781.

[13] Y. Guiard, "Asymmetric division of labor in human skilled bimanual action," J. Motor Behavior, vol. 19, no. 4, pp. 486-517, Feb. 1987.

[14] K. Hinckley, R. Pausch, D. Proffitt, J. Patten, and N. Kassell, "Cooperative bimanual action," in Proc. ACM SIGCHI Conf. Human Factors in Computing Systems, Mar. 1997, pp. 27-34.

[15] R. Suárez, J. Rosell, and N. García, "Using synergies in dual-arm manipulation tasks," in Proc. IEEE Int. Conf. Robotics and Automation, May 2015, pp. 5655-5661.

[16] G. Gioioso, G. Salvietti, M. Malvezzia, and D. Prattichizzo, "Mapping synergies from human to robotic hands with dissimilar kinematics: An approach in the object domain," IEEE Trans. Robotics, vol. 29, no. 4, pp. 825-837, Aug. 2013.

[17] A. Shon, K. Grochow, A. Hertzmann, and R. Rao, Learning shared latent structure for image synthesis and robotic imitation. MIT Press, Dec. 2005, pp. 1233-1240.

[18] I. T. Jolliffe, Principal Component Analysis. Upper Saddle River, NJ, USA: Springer Series in Stat., 2002.

[19] T. Wimböck, B. Jan, and G. Hirzinger, "Synergy-level impedance control for a multifingered hand," in Proc. IEEE/RSJ Int. Conf. Intelligent Robots and Systems, Sept. 2011, pp. 973-979.

[20] W. Chen, C. Xiong, and S. Yue, "Mechanical implementation of kinematic synergy for continual grasping generation of anthropomorphic hand," IEEE/ASME Trans. Mechatronics, vol. 20, no. 3, pp. 1249-1263, June 2015.

[21] J. Romero, T. Feix, C. Ek, H. Kjellström, and D. Kragic, "Extracting postural synergies for robotic grasping," IEEE Trans. Robotics, vol. 29, no. 6, pp. 1342-1352, Dec. 2013.

[22] J. Steffen, R. Haschke, and H. Ritter, "Towards dextrous manipulation using manipulation manifolds," in Proc. IEEE/RSJ Int. Conf. Intelligent Robots and Systems, Sept. 2008, pp. 2738-2743.

[23] J. Rosell, A. Pérez, A. Aliakbar, Muhayyuddin, L. Palomo, and N. García, "The Kautham Project: A teaching and research tool for robot motion planning," in Proc. IEEE Int. Conf. Emerging Technologies and Factory Automation, Sept. 2014.

[24] M. Liarokapis, P. Artemiadis, and K. Kyriakopoulos, "Functional anthropomorphism for human to robot motion mapping," in Proc. IEEE Int. Symp. Robot and Human Interactive Communication, Sept. 2012, pp. 31-36.

[25] I. A. Sucan, M. Moll, and L. E. Kavraki, "The Open Motion Planning Library," IEEE Robotics and Automation Mag., vol. 19, no. 4, pp. 72-82, Dec. 2012.

[26] N. García, J. Rosell, and R. Suárez, "Motion planning using first-order synergies," in Proc. IEEE/RSJ Int. Conf. Intelligent Robots and Systems, Sept. 2015.

[27] Muhayyuddin, A. Akbari, and J. Rosell, "Physics-based motion planning: Evaluation criteria and benchmarking," in Robot 2015: Second Iberian Robotics Conf. Springer, Dec. 2016, pp. 43-55.

[28] M. A. Woodbury, Inverting Modified Matrices, ser. Statistical Research Group Memorandum Reports. Princeton, NJ: Princeton University, 1950, no. 42.

[29] D. A. Harville, Matrix algebra from a statistician's perspective. New York: Springer, Sept. 1997.

[30] M. Fiedler, "Bounds for the determinant of the sum of hermitian matrices," Proc. American Mathematical Society, vol. 30, pp. 27-31, Sept. 1971. 